\title{
Diferentes granulometrias de rações sobre o ganho de peso, crescimento, sobrevivência e reversão sexual para tilápias-do-Nilo (Oreochromis niloticus)
}

\author{
Different diameters of diets about weight gain, growing, survival and sex reversal to Nile \\ tilapia (Oreochromis niloticus)
}

\author{
Lilian Cristina MAKINO ${ }^{1}$; Laura Satiko Okada NAKAGHI ${ }^{1,2}$; Maria do Carmo Faria PAES ${ }^{1}$; Euclides \\ Braga MALHEIROS ${ }^{1,3}$; Teresa Cristina Ribeiro Dias KOBERSTEIN ${ }^{1}$ \\ ${ }^{1}$ Centro de Aquicultura da Universidade Estadual Paulista, Jaboticabal-SP \\ ${ }^{2}$ Departamento de Morfologia e Fisiologia Animal da Faculdade de Ciências Agrárias e Veterinárias da Universidade Estadual \\ Paulista, Jaboticabal-SP \\ ${ }^{3}$ Departamento de Ciências Exatas da Faculdade de Ciências Agrárias e Veterinárias da Universidade Estadual Paulista, Jaboticabal-SP
}

\begin{abstract}
Resumo
O objetivo deste trabalho foi avaliar os efeitos de três diferentes granulometrias de rações fareladas $(0,25,0,35$ e 0,50 $\mathrm{mm}$ ) sobre o ganho de peso, crescimento, sobrevivência e proporção sexual para larvas de tilápia-do-Nilo (Oreochromis niloticus) aos 30, 60 e 90 dias de idade. Nos primeiros 30 dias de vida, as larvas foram revertidas com rações contendo hormônio 17 a-metiltestosterona na dosagem de $60 \mathrm{mg} / \mathrm{kg}$ de ração, respeitando-se as diferentes granulometrias e após este período, foram medidas, pesadas e contadas para cálculo da taxa de sobrevivência. Para as variáveis biométricas de peso e comprimento total, foi utilizado o delineamento inteiramente casualizado (DIC), em parcelas subdivididas, com três tratamentos e três repetições, considerando-se parcelas as três granulometrias de ração e subparcelas as três idades. Ao término do estudo, os peixes alimentados com as granulometrias 0,35 e $0,25 \mathrm{~mm}$, obtiveram os melhores resultados para o ganho de peso $(14,42 \mathrm{~g}$ e $13,36 \mathrm{~g})$ e comprimento total $(83,13 \mathrm{~mm}$ e $82,72 \mathrm{~mm})$, não diferindo estatisticamente e as rações com diâmetros de grão 0,50 e 0,35 mm resultaram em 96,66\% e 93,33\% de machos, respectivamente, após análise histológica das gônadas, sem diferenças estatísticas entre os tratamentos.
\end{abstract}

Palavras-chave: Oreochromis niloticus. Alimentação. Diâmetro de ração. Larvas.

\begin{abstract}
The aim of this work was to evaluate the effects of three different diameters of crumble diets $(0.25,0.35$ and $0.50 \mathrm{~mm})$ on weight gain, growing, survival and sexual proportion to Nile tilapia (Oreochromis niloticus) larvae with 30, 60 and 90 days of age. In the first 30 days of age larvae were submitted to sex reversion with diets containing the hormone 17 a-methyltestosterone in a dosage of $60 \mathrm{mg} / \mathrm{kg}$ of ration, respecting the different diameters and after this period, larvae were measured, weighed and counted for calculation of survival taxes. For the biometrical parameters as weight and total length a completely randomized design was used in subdivided installments with three treatments and three repetitions, considering installments the three diameters of diet and the sub installments, the three ages. In the end of this study, fish fed with diets of grain diameters 0.35 and $0.25 \mathrm{~mm}$ obtained the best results for weight gain (14.42 $\mathrm{g}$ and $13.36 \mathrm{~g})$ and total length $(83.13 \mathrm{~mm}$ and $82.72 \mathrm{~mm})$, although these results were not statistically significant, and diets with grain diameters 0.50 and $0.35 \mathrm{~mm}$ resulted in $96,66 \%$ and $93,33 \%$ of males, respectively, after histological analysis of gonads, without statistics differences between the treatments.
\end{abstract}

Keywords: Oreochromis niloticus. Feeding. Milling degree. Larvae.

\section{Introdução}

A tilápia-do-Nilo, Oreochromis niloticus, é a espécie de água doce mais utilizada nos cultivos comerciais, principalmente por sua rusticidade, rápido crescimento, carne de ótima qualidade e boa aceitação pelo mercado de lazer (pesque-pague) e alimentício (frigoríficos) pelas qualidades nutritivas e organolépticas do seu filé1,2,3.
Correspondência para:

Lilian Cristina Makino

Departamento de Morfologia e Fisiologia Animal da Faculdade de Ciência Agrárias e Veterinárias da UNESP, Campus de Jaboticabal - SP. Av. Via de Acesso Prof. Paulo Donato Castellane, s/n, 14884-900, Jaboticabal - SP, Brasil e-mail: liliancrism02@yahoo.com.br

Recebido: $12 / 07 / 2007$ Aprovado: 10/06/2010

Agradecimento: Ao CNPq pelo auxílio concedido em forma de bolsa de mestrado 
Para a maioria das espécies de peixes, a utilização de dietas artificiais como fonte de alimento durante a fase de larvicultura não apresenta bons resultados devido à imaturidade do aparelho digestório das larvas ${ }^{4}$. Contudo, para a tilápia-do-Nilo, tal fato possui pouca relevância, pois mesmo no período larval esta já se alimenta com ração e se desenvolve bem, apresentando bons índices de crescimento e ganho de peso ${ }^{3}$, tornando-se possível nesta fase da vida, a reversão do sexo pela adição de um hormônio masculinizante à ração (17 $\alpha$-metiltestosterona), o qual é fornecido aos peixes durante o primeiro mês de vida ${ }^{3,5}$. Trabalhos de diversos autores ${ }^{3,6,7,8}$ comprovaram que a melhor forma de se alimentar tilápias-do-Nilo nas fases larval e juvenil é com ração farelada, apresentando os melhores índices de crescimento e ganho de biomassa quando alimentadas com rações de diâmetros entre 0,35 e $0,50 \mathrm{~mm}$.

O presente trabalho desenvolvido com larvas de tilápia-do-Nilo (Oreochromis niloticus) submetidas ao processo de reversão sexual e a diferentes granulometrias de ração teve o intuito de avaliar seus efeitos sobre o ganho de peso, crescimento, sobrevivência e proporção sexual dos peixes aos 30, 60 e 90 dias de idade.

\section{Material e Método}

O trabalho foi realizado no Departamento de Morfologia e Fisiologia Animal da FCAV/UNESP e no Setor de Tilapicultura do Centro de Aquicultura da UNESP, ambos localizados em Jaboticabal/SP, por um período de 90 dias. Para tal, foram utilizadas 900 larvas de tilápias-do-Nilo com dois dias de idade póseclosão (DPE), distribuídas em um delineamento experimental inteiramente casualizado (DIC), com três tratamentos e três repetições em um esquema de parcelas subdivididas, tendo como parcelas as três granulometrias de ração e subparcelas as três idades analisadas. Os tratamentos foram constituídos pelas granulometrias de ração 0,25, 0,35 e 0,50 mm.
Para a realização do experimento, utilizaram-se nove aquários de 130 litros, equipados com sistemas de aeração e abastecimento hídrico contínuos. A vazão da mesma foi de $400 \mathrm{~mL} / \mathrm{min}$, possibilitando $100 \%$ de renovação a cada duas horas. Os aquários foram sifonados diariamente pela manhã e à tarde para eliminação dos resíduos. As temperaturas da água (máxima e mínima) eram aferidas diariamente pela manhã, antes da sifonagem dos mesmos. Os outros parâmetros físico-químicos analisados no início do experimento foram $\mathrm{pH}$, oxigênio dissolvido, alcalinidade, dureza e amônia.

A ração utilizada no experimento, cuja composição está representada na tabela 1 , era inicialmente extrusada, que posteriormente foi triturada em moinho de faca no diâmetro de grão de $0,70 \mathrm{~mm}$, sendo depois peneirada manualmente de maneira enérgica em três peneiras, com as respectivas granulometrias a serem testadas, que foram: $0,25,0,35$ e $0,50 \mathrm{~mm}$. No momento da preparação das rações adicionou-se o andrógeno 17 a-metiltestosterona na quantidade de 60 $\mathrm{mg} / \mathrm{kg}$ de ração, diluído em $500 \mathrm{~mL}$ de álcool etílico.

As larvas começaram a ser alimentadas com as rações tratamento (granulometrias: 0,25; 0,35 e 0,50 $\mathrm{mm}$ ) com dois dias após a eclosão, sob o regime ad libitum, na frequência de três vezes ao dia $(9 \mathrm{~h} 00 \mathrm{~min}$, 14h00min e $17 \mathrm{~h} 30 \mathrm{~min}$ ), durante 30 dias, período no qual se realiza o manejo da reversão sexual para esta espécie de peixe. Após esse período, utilizou-se a granulometria $0,35 \mathrm{~mm}$ (granulometria intermediária) sem hormônio para o crescimento e mantença dos peixes até os 90 dias de idade, com o mesmo regime de alimentação.

Após 30 dias de alimentação com as rações tratamento, período que compreendeu o término do processo do manejo de reversão sexual das larvas, os peixes foram contados para o cálculo da taxa de sobrevivência. A sobrevivência foi calculada utilizando-se a fórmula: $\mathrm{TS}=\mathrm{N}^{\circ}$. de peixes vivos/ $\mathrm{N}^{\circ}$. total de peixes X 100 . 
Tabela 1 - Composição da ração experimental - Jaboticabal - 2004

\begin{tabular}{|c|c|c|c|}
\hline Ingredientes & Quantidade (\%) & Componente & Quantidade \\
\hline Milho & 5,0 & Proteína Bruta (\%) & 35,0 \\
\hline Farelo de Trigo & 29,0 & Fibra Bruta (\%) & 5,19 \\
\hline Farelo de Arroz & 5,0 & Extrato Etéreo (\%) & 6,22 \\
\hline Farelo de Soja & 39,0 & $\begin{array}{l}\text { Extrato Não- } \\
\text { Nitrogenado (\%) }\end{array}$ & 31,52 \\
\hline Farinha de Peixe & 21,0 & Matéria Seca (\%) & 88,28 \\
\hline Suplemento vit. min. ${ }^{1}$ & 1,0 & Energia Bruta (Kcal/kg) & 4130 \\
\hline Total & 100,0 & & \\
\hline
\end{tabular}

As amostragens dos peixes foram realizadas aos 30, 60 e 90 dias de idade, sendo os animais eutanasiados em solução de benzocaína a $0,1 \%$ e fixados inteiros em solução de formol tamponado a $10 \% \mathrm{com} \mathrm{pH}=$ 7,4 , durante 24 horas. Foram coletados 30 peixes/tratamento de cada idade proposta. Os mesmos foram analisados quanto ao ganho de peso (g) e comprimento total $(\mathrm{mm})$ e análise histológica das gônadas ${ }^{9}$ ao final de 90 dias, quando estas permitem um diagnóstico mais eficaz com relação ao sexo dos peixes.

Variáveis biométricas de peso (g), comprimento total (mm) e da taxa de sobrevivência (\%) foram submetidas à análise de variância (ANOVA). Nos casos onde houve diferenças significativas foi aplicado o teste de Tukey $(\alpha=5 \%)$. Para análise comparativa entre as frequências obtidas para machos, fêmeas e intersexos foi aplicado o Teste Exato de Fisher, adotando-se o critério de significância ao nível de $\alpha=5 \%$. As análises estatísticas foram feitas por meio do programa estatístico SAS (Statistic Analysis System), versão 8.0 ${ }^{10}$.

\section{Resultados}

Os valores dos parâmetros físico-químicos da água nos tanques experimentais, tais como temperatura, $\mathrm{pH}$, oxigênio dissolvido, alcalinidade, dureza e amônia foram monitorados e seus valores médios foram respectivamente, $25,9 \pm 1,36^{\circ} \mathrm{C}$ a $28,0 \pm 0,62{ }^{\circ} \mathrm{C}, 7,7$ $\pm 0,1,8,8 \pm 0,5 \mathrm{mg} / \mathrm{L}, 146 \pm 11 \mathrm{mg} / \mathrm{L}, 34 \pm 5 \mathrm{mg} / \mathrm{L} \mathrm{e}$ $0,12 \pm 0,1 \mathrm{mg} / \mathrm{L}$.

Os valores de $\mathrm{F}$ e os coeficientes de variação $(\mathrm{CV})$ obtidos na análise de variância para o peso e comprimento total estão representados na tabela 2 . Na tabela 3 constam os valores médios obtidos para o peso, comprimento total e as taxas de sobrevivência.

Tabela 2 - Análise de variância: Estatísticas F e coeficientes de variação $(\mathrm{CV})$ para as variáveis peso (g) e comprimento total (mm) - Jaboticabal - 2005

\begin{tabular}{ccc}
\hline Estatísticas & Peso $(\mathrm{g})$ & Comprimento Total $(\mathbf{m m})$ \\
\hline F para Tratamento & $2,36^{\mathrm{NS}}$ & $2,40^{\mathrm{NS}}$ \\
F para Idade & $158,41^{*}$ & $436,78^{\star}$ \\
CV (parcelas) & $77,32 \%$ & $20,29 \%$ \\
CV (subparcelas) & $63,55 \%$ & $19,19 \%$ \\
\hline
\end{tabular}

* Significativo para $\mathrm{P}<0,05$, NS para $\mathrm{P}>0,05=$ valores não significativos e CV = coeficiente de variação 
Tabela 3 - Valores médios obtidos para as variáveis peso $(\mathrm{g})$ e comprimento total $(\mathrm{mm})$ aos 30, 60 e 90 dias de idade e da sobrevivência das larvas de tilápias-do-Nilo (\%) até os 30 dias, referentes aos tratamentos (granulometrias de 0,25, 0,35 e 0,50 $\mathrm{mm}$ ) - Jaboticabal - 2005

\begin{tabular}{lcccc}
\hline Variáveis & Idades & \multicolumn{3}{c}{ Tratamentos } \\
\cline { 3 - 5 } & & $\mathbf{0 , 2 5} \mathbf{~ m m}$ & $\mathbf{0 , 3 5} \mathbf{~ m m}$ & $\mathbf{0 , 5 0 ~} \mathbf{~ m}$ \\
\hline Peso (g) & 30 dias & $0,55^{\mathrm{c}}$ & $0,64^{\mathrm{c}}$ & $0,54^{\mathrm{c}}$ \\
& 60 dias & $3,96^{\mathrm{b}}$ & $8,53^{\mathrm{b}}$ & $5,39^{\mathrm{b}}$ \\
& 90 dias & $13,36^{\mathrm{a}}$ & $14,42^{\mathrm{a}}$ & $10,91^{\mathrm{a}}$ \\
\hline $\begin{array}{l}\text { Comprimento } \\
\text { Total (mm) }\end{array}$ & 30 dias & $31,65^{\mathrm{c}}$ & $32,15^{\mathrm{c}}$ & $32,24^{\mathrm{c}}$ \\
& 60 dias & $49,08^{\mathrm{b}}$ & $64,84^{\mathrm{b}}$ & $62,76^{\mathrm{b}}$ \\
\hline $\begin{array}{l}\text { Sobrevivência } \\
\text { (\%) }\end{array}$ & 90 dias & $82,72^{\mathrm{a}}$ & $83,13^{\mathrm{a}}$ & $76,36^{\mathrm{a}}$ \\
\hline
\end{tabular}

*As médias seguidas por letras diferentes (nas colunas) diferem entre si para valores de $\mathrm{P}<0,05$ (Teste de Tukey)

Os valores médios para peso e comprimento total mostram que não houve diferenças estatísticas significativas (Tabela 2).

Os dados da tabela 3 mostraram que os valores médios de peso e comprimento total aos 30, 60 e 90 dias de idade diferiram significativamente entre eles. O peso e o comprimento não variaram significativamente aos 30 dias de idade para as três granulometrias, sendo a mesma observação válida para as taxas de sobrevivência.

No tocante às proporções entre os sexos, as rações com diâmetros de grão de 0,50 e $0,35 \mathrm{~mm}$, foram as que apresentaram os melhores resultados com frequências relativas entre $96,66 \%$ e $93,33 \%$ de machos, respectivamente (Tabela 4). O teste estatístico de Fisher aplicado nesta análise foi não significativo para valores de $\mathrm{P}>$ 0,05 , indicando que o sexo dos peixes e os tratamentos são eventos independentes (Tabela 5).

\section{Discussão}

As variáveis físico-químicas registradas para a água ficaram dentro dos valores preconizados para a aquicultura e para o cultivo da espécie $e^{11,12}$.

Tabela 4 - Frequência absoluta (fa) e relativa (fr) de machos, fêmeas e intersexos referentes às granulometrias de ração testadas $(0,25,0,35$ e $0,50 \mathrm{~mm})$ aos 90 dias de idade para larvas de tilápias-do-Nilo - Jaboticabal - 2005

\begin{tabular}{cccccccc}
\hline Granulometria & $\begin{array}{c}\mathbf{N}^{\mathbf{0}} \text {. de } \\
\text { peixes }\end{array}$ & \multicolumn{2}{c}{ Machos } & \multicolumn{2}{c}{ Fêmeas } & \multicolumn{2}{c}{ Intersexos } \\
\hline & & fa (n) & fr (\%) & fa (n) & fr (\%) & fa (n) & fr (\%) \\
$\mathbf{0 , 2 5} \mathbf{~ m m}$ & 30 & 25 & 83,33 & 4 & 13,33 & 1 & 3,33 \\
$\mathbf{0 , 3 5} \mathbf{~ m m}$ & 30 & 28 & 93,33 & 2 & 6,66 & 0 & 0,00 \\
$\mathbf{0 , 5 0} \mathbf{~ m m}$ & 30 & 29 & 96,66 & 1 & 3,33 & 0 & 0,00 \\
\hline
\end{tabular}

Tabela 5 - Valores obtidos pelo Teste Exato de Fisher referente à proporção entre os sexos Jaboticabal - 2005

\begin{tabular}{ll}
\hline Teste Exato de Fisher & $0,02(\mathrm{P}=0,56)^{*}$ \\
* Teste não significativo para valores de $\mathrm{P}>0,05$
\end{tabular}


Com relação aos dados obtidos na tabela 2, os altos valores de CV encontrados para o peso e comprimento total em todos os tratamentos e idades eram esperados, pois os autores ${ }^{1,13}$ afirmaram que o crescimento heterogêneo entre larvas e juvenis de tilápias é uma característica comum, estando relacionada ao estabelecimento de hierarquias entre os peixes, em que há poucos animais grandes e muitos animais pequenos. Desta maneira, os índices de crescimento e ganho de peso dos peixes ao término do período de reversão sexual podem apresentar uma grande variabilidade, como foi encontrado neste trabalho. $\mathrm{O}$ crescimento e a taxa de sobrevivência das larvas durante o tratamento hormonal dependem de vários fatores, entre eles densidade de estocagem, alimentação e temperatura ${ }^{14,15}$. Além disto, os indivíduos dominantes dentro de uma população podem consumir mais alimento e crescerem mais rapidamente, deixando menos alimento para os demais com comportamento mais submisso, que acabam por apresentar menor crescimento tornando-se, portanto, mais vulneráveis ao canibalismo ${ }^{15,16}$. Ressalta-se que as taxas elevadas de sobrevivência encontradas neste experimento se mostraram superiores à taxa proposta como normal para o período de reversão sexual por autores ${ }^{11}$ que propuseram uma taxa de sobrevivência ideal de $70 \%$ a $80 \%$. No presente trabalho, não houve diferenças quanto ao ganho de peso, crescimento e sobrevivência entre os tratamentos, sendo assim, produtores de tilápias podem fazer uso de rações com qualquer uma das granulometrias propostas sem prejuízo ao desempenho e sobrevivência das larvas.

A eficiência da digestão dos alimentos, como mostrado na tabela 3 está relacionada com a superfície de exposição das partículas alimentares às secreções digestivas, superfície esta que é inversamente proporcional ao grau de moagem e ao trânsito gastrintestinal ${ }^{7,17,18}$. De acordo com os autores ${ }^{3,4,7,19,20}$ durante o processamento das rações o grau de moagem dos seus ingredientes mostrou-se como um dos fatores de maior importância, pois quanto mais finamente moídos, maiores seriam os custos de produção, devido ao maior consumo de energia e ao tempo de moagem. Mediante a moagem dos ingredientes inferior a $0,50 \mathrm{~mm}$, pode-se inferir que apesar da eficiência da digestão dos alimentos melhorar com a redução do tamanho da partícula ${ }^{17}$, concomitantemente poderá ocorrer uma redução nas taxas de trânsito alimentar pelo trato digestório, ${ }^{4,7,19,20}$. Este fato pode levar à limitação da ingestão voluntária de alimento pelo animal, acarretando em prejuízos ao desempenho, como a redução das taxas de crescimento e aumento de peso $^{2}$. Podem-se acrescentar as observações de Pezzato et al. ${ }^{8}$ no tocante à perda de nutrientes por lixiviação na superfície do corpo d'água, aumentando a carga poluente no mesmo e naquele que recebe os efluentes. Contrariamente aos autores acima, o presente experimento não apresentou resultados significativos com relação às granulometrias analisadas nos parâmetros estudados (peso, comprimento total e sobrevivência).

Quanto aos resultados de reversão sexual, como não foram encontrados trabalhos relacionando taxas de reversão sexual com rações de diferentes granulometrias contendo andrógenos, sugere-se que o grau de incorporação de hormônio masculinizante à superfície da ração seja proporcional ao diâmetro de grão da mesma.

As implicações de um processamento inadequado dos ingredientes componentes das rações para a tilápia nilótica durante a fase de reversão sexual podem afetar a produção tanto no aspecto produtivo (juvenis com menores pesos e tamanhos), quanto no aspecto financeiro, pois a moagem muito fina dos ingredientes torna oneroso o processo produtivo com maiores gastos de energia e de mão de obra ${ }^{7}$.

\section{Conclusão}

Diante dos resultados obtidos, conclui-se que tilápias alimentadas com rações de diferentes granulometrias não são afetadas quanto ao ganho de peso, comprimento total, sobrevivência e efetividade da reversão sexual. 


\section{Referências}

1. BORGES, A. M.; MORETTI, J. O. C.; MCMANUS, C.; MARIANTE, A. S. Produção de populações monossexo macho de tilápia-do-nilo da linhagem Chitralada. Pesquisa Agropecuária Brasileira, v. 40, n. 2, p. 153-159, 2005.

2. MEURER, F.; HAYASHI, C.; BOSCOLO, W. R. Lipídeos na alimentação de alevinos revertidos de tilápia-do-nilo (Oreochromis niloticus, L.). Revista Brasileira Zootecnia, v. 31, n. 2, p. 566-573, 2002.

3. MEURER, F.; HAYASHI, C.; BOSCOLO, W. R. Influência do processamento da ração no desempenho e sobrevivência da tilápia-do-nilo durante a reversão sexual. Revista Brasileira Zootecnia, v. 32, n. 2, p. 262-267, 2003.

4. HAYASHI, C.; BOSCOLO, W. R.; SOARES, C. Uso de diferentes graus de moagem dos ingredientes em dietas para a tilápia-do-nilo (Oreochromis niloticus L.) na fase de crescimento. Acta Scientiarum, v. 21, n. 3, p. 733-737, 1999.

5. PHELPS, R. P.; SALAZAR, G. C.; ABE, V.; ARGUE, B. Sex reversal and nursery growth of Nile tilapia, (Oreochromis niloticus), free-swimming in earthen ponds. Aquaculture Research, v. 26, p. 293-295, 1995.

6. BOMBARDELLI, R. A.; HAYASHI, C.; MEURER, F.; FORNARI, D. C. Avaliação de rações fareladas e micropeletizadas para larvas de tilápia-do-nilo (Oreochromis niloticus) - desempenho e efetividade da reversão sexual. Acta Scientiarum, v. 26, n. 2, p. 197-201, 2004.

7. MEURER, F.; BOMBARDELLI, R. A.; HAYASHI, C.; FORNARI, D. C. Grau de moagem dos alimentos em rações para a tilápia-do-nilo (Oreochromis niloticus) durante o período de reversão sexual. Acta Scientiarum, v. 27, n. 1, p. 81-85, 2005.

8. PEZZATO, L. E.; MIRANDA, E. C.; FURUYA, W. M.; PINTO, L. G. Q.; BARROS, M. M.; ROSA, G. J. M. Diâmetro do ingrediente e a digestibilidade aparente de rações por duas espécies de peixes tropicais. Acta Scientiarum, v. 24, n. 4, p. 901- 907, 2002.

9. CARVALHO, E. D.; FORESTI, F. Reversão de sexo em tilápiado-nilo, Oreochromis niloticus TREWAVAS, 1983, induzida por 17-alfa-metiltestosterona: proporção de sexo e histologia das gônadas. Revista Brasileira de Biologia, v. 56, n. 2, p. 249-262, 1996.

10.STTISTICAL ANALISYS SYSTEM. SAS user's guide: statistics. Versão 8.0. Cary: SAS, 2004.
11. POPMA, T. J.; LOVSHIN, L. Worldwide prospects for commercial production of tilapia. Alabma: International Center for Aquaculture and Aquatic Environments, 1996. 23 p. (Research and Development, Series n. 41).

12.TACHIBANA, L.; CASTAGNOLLI, N.; PEZZATO, L. E.; BARROS, M. M.; VALLE, J. B.; SIQUEIRA, M. Desempenho de diferentes linhagens de tilápia-do-nilo (Oreochromis niloticus) na fase de reversão sexual. Acta Scientiarum, v. 26 , n. 3, p. 305- 311, 2004.

13. VOLPATO, G. L.; FRIOLI, P. M. A.; CARRIERI, M. P. Heterogeneous growth in fish: some data in the Nile tilapia Oreochromis niloticus and a general view about causal mechanisms. Boletim de Fisiologia Animal, v. 13, p. 7-22, 1989.

14.BOCEK, A.; PHELPS, R. P.; POPMA, T. J. Effect of feeding frequency on sex-reversal and on growth of Nile tilapia, Oreochromis niloticus. Journal of Applied Aquaculture, v. 1, n. 3, p. 97-103, 1992.

15. MAINARDES-PINTO, C. S. R.; VERANI, N. F.; CAMPOS, B. E. S.; SILVA, A. L. Masculinização da tilápia-do-nilo, Oreochromis niloticus, utilizando diferentes rações e diferentes doses de 17 a-metiltestosterona. Revista Brasileira Zootecnia, v. 29, n. 3, p. 654-659, 2000.

16. VERA CRUZ, E. M.; MAIR, G. C. Conditions for effective androgen sex-reversal in Oreochromis niloticus (L.). Aquaculture, v. 22, n. 2, p. 237-248, 1994.

17. MONTICELLI, C. J.; MENTEN, J. F. M.; ZANOTTO, D. L.; LIMA, G. J. M. M.; GUIDONI, A. L. Efeito da granulometria do milho, da área por animal e do sexo sobre o desempenho de suínos em crescimento e terminação. Revista Brasileira Zootecnia, v. 25, n. 6, p. 1150-1162, 1996.

18. SOARES, C. M.; HAYASHI, C.; BOSCOLO, W. R.; MEURER, F. Diferentes graus de moagem dos ingredientes em dietas peletizadas para a tilápia-do-nilo (Oreochromis niloticus, L.) em fase de crescimento. Desempenho e digestibilidade aparente. Zootecnia Tropical, v. 21, n. 3, p. 275-287, 2003.

19. NATIONAL RESEARCH COUNCIL (NCR). Nutrient requirement of fish. Washington, DC: National Academy Press, 1993.

20.ZANOTTO, D. L.; NICOLAIEWSKY, S.; FERREIRA, A. S. Granulometria do milho na digestibilidade das dietas para suínos em crescimento e terminação. Revista Brasileira Zootecnia, v. 24, n. 3, p. 428-436, 1995. 